\title{
Transcriptomic and Histological Analysis of Exposed Facial Skin Areas Wrinkled or Not and Unexposed Skin
}

Renan Martin ( $\nabla$ renan.paulo.martin@gmail.com )

Johns Hopkins University School of Medicine https://orcid.org/0000-0002-6747-8784

Patricia Varela

Universidade Federal de Sao Paulo

Caio Peres Gomes

Universidade Federal de Sao Paulo

Maryana Mara Marins

Universidade Federal de Sao Paulo

Rafael Filippelli-Silva

Universidade Federal de Sao Paulo

Samira Yarak

Universidade Federal de Sao Paulo

Juliana L.M. Soares

Universidade Federal de Sao Paulo

Adriana Sanudo

Universidade Federal de Sao Paulo

Jolanta Idkowiak-Baldys

Avon Products Inc

Siming Chen

Avon Products Inc

Cheng Hwang

Avon Products Inc

Yong Zhuang

Avon Products Inc

John Lyga

Avon Products Inc

João Bosco Pesquero

Universidade Federal de Sao Paulo

\section{Ediléia Bagatin}

Universidade Federal de Sao Paulo https://orcid.org/0000-0001-7190-8241 


\section{Research Article}

Keywords: environmental and hormonal factor, RNASeq, aminoacids, photo-exposed, phototype III

Posted Date: September 22nd, 2021

DOI: https://doi.org/10.21203/rs.3.rs-234540/v2

License: (c) (i) This work is licensed under a Creative Commons Attribution 4.0 International License. Read Full License

Version of Record: A version of this preprint was published at Molecular Biology Reports on December 1st, 2021. See the published version at https://doi.org/10.1007/s11033-021-06973-y. 


\section{Abstract}

Skin aging involves genetic, environmental and hormonal factors. Facial wrinkles also depend on muscular activity. Gene expression investigation may be useful for new anti-aging products. To evaluate structure and gene expression differences among exposed and unexposed skin in menopausal women. Cross-sectional study, including 15 menopausal women, 55-65yo, phototype III; photo-exposed, periorbital wrinkles (A1), preauricular, not wrinkled (A2), and unexposed gluteal (A3) areas were described and compared by non-invasive measures, histology, immunohistochemistry and gene expression (RNASeq); participants mean age was 61yo, presenting moderate periorbital wrinkles and light facial photodamage. Higher roughness, wrinkles number and echogenicity were observed in A1 and A2 versus A3. Decreased epidermal thickness and dermal collagen IV were demonstrated in A1 versus A2 and A3. Exposed areas impacted different pathways compared to unexposed. Exposed wrinkled skin (A1) showed impact on cell movement with decreased inflammatory activation state. Pathways related to lipid and aminoacids metabolism were modulated in non-wrinkled exposed (A2) compared to unexposed (A3) skin. Expected histological findings and gene expression differences among areas were observed. Photoaging in menopausal women may modulate lipid and aminoacids metabolism and decrease inflammatory and keratinization pathways, cellular homeostasis, immune response, fibrogenesis and filament formation. These findings may help development of new therapies for skin health and aging control.

\section{Introduction}

Skin aging is a complex biological process caused by intrinsic or genetic and extrinsic or environmental factors $[28,47]$. The superposition of intrinsic and extrinsic aging, named photoaging, is responsible for $85 \%$ of aged skin phenotype in exposed areas. The chronic exposure to aggressors like solar radiation, especially ultra-violet (UV), tobacco, pollution and hot climate causing activation of transient receptor potential cation channel subfamily $\vee$ member 1 (TrpV1), accelerate the skin aging $[11,30]$. Additionally, forehead, eyes, and lip areas are affected by superposition of dynamic wrinkles related to muscular activity [31].

Clinical signs of aged skin include reduced thickness, hydration and elasticity, with progressive development of sagginess, wrinkles, sulcus, and dyschromia [35], which are more evident and severe in photo-exposed areas of Caucasian population [20]. Photo-exposure causes skin damage due to DNA mutations, particularly at mitochondrial level, increased formation of reactive oxygen species (ROS), synthesis and release of pro-inflammatory mediators. Furthermore, synthesis of dermal matrix components, especially collagen I and IV (present in the dermal-epidermal junction - DEJ) and elastin is reduced with concomitant increase in their degradation, by metalloproteinases (MMPs). Finally, antioxidant depletion, lipid peroxidation and decomposition of sebaceous lipids also occur [3, 36, 37, 40, $47,48]$.

Skin epidermis comprises four layers: basal, spinous, granular, and cornified. The stratum corneum, essential for cutaneous barrier, is composed of corneocytes and lipids, which are responsible for water 
retention, maintaining hydration and protecting our body from desiccation [12]. The cutaneous barrier also protects the skin from external damage through the presence of sebum, which also has antimicrobial properties [36]. Aging process has been associated with changes in lipid metabolism altering skin barrier function, including synthesis and processing of stratum corneum lipids [12]. Increasing age promotes a decrease in skin lipid levels, especially ceramides [25, 41]. Furthermore, aging promotes gradual lipoperoxidation of skin structures. The lipid peroxidation of polyunsaturated fatty acids due to oxidative stress causes chromatin damage, such as histones methylation and acetylation. The chromatin modifications constitute important mechanism for regulating expression of genes involved in cell maintenance and longevity [38]. Skin lipids can also be oxidized by UV radiation and mediate inflammatory responses worsening skin aging.

Lipidic and caloric restrictions suppress the MTOR pathway activating the autophagy and antioxidant response, which control the aging process [14]. The role of other dietary factors has been highlighted [7]. In women, besides environmental aggressors, menopause is an additional aging factor, as the decrease in estrogen signaling has pronounced effect on skin [6]. Furthermore, menopause also promotes an adverse lipid profile [29].

Skin care depends on adequate cleansing, hydration and photoprotection to maintain the cutaneous barrier integrity [4]. Beyond aesthetic benefits, it is possible to prevent the development of pre-neoplastic lesions and non-melanoma skin cancer by daily use of broad-spectrum sunscreens, mainly in specific risk groups (fair skin, occupational exposition, kidney transplant recipients under chronic immune suppression, some genodermatosis, like xeroderma pigmentosum, and more)[2, 23, 42]. The idea of reversing skin aging signs has motivated the introduction of a huge variety of cosmeceuticals in the market, usually safe but often lacking sufficient evidence of efficacy based on randomized and wellcontrolled clinical trials $[4,33]$. They are non-prescription products, neither drugs nor real cosmetics, but capable of modulating skin biological functions $[1,16,17]$ and should be added to therapeutic regimen for prevention of skin cancer recurrence [21]. Progress in formulations has been made in recent years, looking for better skin penetration and activity [39].

The clinical and therapeutic aspects of facial wrinkles and skin aging have been widely reported. However, genomic aspects are less investigated despite the fact that skin is a useful organ to identify epigenomic changes along with assessment of aging profile by transcriptomics comparing photoexposed and non-photo-exposed areas. Thus, our aim was to confirm histological features and analyze gene expression profiles of the periorbital (crow's feet) and preauricular skin (wrinkle-free), both photoexposed areas and compare them to non-photo-exposed skin from gluteal region, in menopausal women. Molecular studies, including genomics and transcriptomics, can be used for development of a translational approach to skin care, increasing its efficacy. We believe our results may shed light into the development of future therapeutic strategies for skin health, as well as for promoting lifestyle changes that would aid aging prevention and control.

\section{Results}


The study population mean age was 61, phototype III, with moderate periorbital dynamic wrinkles and signs of facial photoaging, i.e., light sagginess, wrinkles at rest and dyschromia.

\section{Non-invasive instrumental measures}

Results from non-invasive instrumental methods (profilometry and high frequency ultrasound) and comparisons between areas (photo-exposed: A1, periorbital and A2, preauricular; non-photo-exposed: A3, gluteal region) are presented in Table 1 and Table 2. The most relevant findings were: A1 was smoother than A2; higher roughness, wrinkles number and echogenicity in A1 and A2 compared to A3.

Table 1

Mean \pm standard deviation of each variable of skin relief (Visioscan VC $98 \AA$ ) and dermal echogenicity (20 MHz ultrasound DermaScan (B) in the three areas

\begin{tabular}{|lllllll|}
\hline & $\begin{array}{l}\text { SEsm } \\
\text { smoothness }\end{array}$ & $\begin{array}{l}\text { Ser } \\
\text { roughness }\end{array}$ & $\begin{array}{l}\text { SEsc } \\
\text { scaliness }\end{array}$ & $\begin{array}{l}\text { Sew } \\
\text { wrinkles }\end{array}$ & Echogenicity & TOTAL \\
\hline Area 1 & $76.1 \pm 23.3$ & $5.9 \pm 2.0$ & $0.744 \pm 0.320$ & $54.3 \pm 15.2$ & $0.524 \pm 0.092$ & $1.32 \pm 0.17$ \\
\hline Area 2 & $59.9 \pm 9.3$ & $5.1 \pm 1.4$ & $0.602 \pm 0.222$ & $44.2 \pm 7.6$ & $0.566 \pm 0.092$ & $1.34 \pm 0.18$ \\
\hline Area 3 & $68.4 \pm 13.4$ & $3.6 \pm 1.0$ & $0.549 \pm 0.111$ & $56.5 \pm 10.1$ & $0.447 \pm 0.106$ & $1.05 \pm 0.37$ \\
\hline p-value & 0.004 & $<0.001$ & 0.063 & 0.007 & 0.001 & 0.003 \\
\hline
\end{tabular}

$\mathrm{A} 1$ = photo-exposed periorbital area; $\mathrm{A} 2$ = photo-exposed preauricular area; $\mathrm{A} 3$ = non-photo-exposed gluteal area; SEsm - the lower, the smoother; SEr - the lower, the rougher; SEsc - the lower, less desquamation; Sew - the lower, less wrinkles 
Table 2

Pairwise comparisons among areas for each variable of skin relief and dermal echogenicity (mean \pm standard error, ONEWAY-ANOVA followed by multiple comparisons, with Bonferroni correction)

\begin{tabular}{|llll|}
\hline & A1 vs A2 & A1 vs A3 & A2 vs A3 \\
\hline SEsm & $16.2 \pm 4.8$ & $7.7 \pm 4.8$ & $-8.5 \pm 4.8$ \\
smoothness & $(p=0.001)$ & $(p=0.113)$ & $(p=0.079)$ \\
Ser & $0.8 \pm 0.5$ & $2.3 \pm 0.5$ & $1.5 \pm 0.5$ \\
roughness & $(p=0.134)$ & $(p<0.001)$ & $(p=0.005)$ \\
\hline SEsc & $0.142 \pm 0.086$ & $0.195 \pm 0.086$ & $0.053 \pm 0.086$ \\
scaliness & $(p=0.097)$ & $(p=0.023)$ & $(p=0.538)$ \\
Sew & $10.1 \pm 4.2$ & $-2.2 \pm 4.2$ & $-12.3 \pm 4.2$ \\
wrinkles & $(p=0.015)$ & $(p=0.594)$ & $(p=0.003)$ \\
Echogenicity & $-0.042 \pm 0.032$ & $0.077 \pm 0.032$ & $0.119 \pm 0.032$ \\
& $(p=0.194)$ & $(p=0.018)$ & $(p<0.001)$ \\
\hline TOTAL & $-0.02 \pm 0.09$ & $0.27 \pm 0.09$ & $0.29 \pm 0.09$ \\
& $(p=0.815)$ & $(p=0.004)$ & $(p=0.002)$ \\
\hline
\end{tabular}

\section{Histology and immunohistochemistry}

Histological and immunohistochemical main findings were the decreased epidermal thickness and dermal collagen IV expression in periorbital wrinkles compared to preauricular skin as well as when both were compared to gluteal region. Figure 1 shows in the first row, images from exposed crows' feet area (A1) showing thin, atrophic epidermis with deep ridges (H\&E); mild degree of dermal elastosis, compared with preauricular area (Masson's trichrome) and less marked collagen IV, compared to preauricular area (anti-collagen IV antibody); in the second row, the images from preauricular area, exposed and wrinklefree (A2) shows thin, atrophic and rectified epidermis (H\&E); higher degree of dermal elastosis (Masson's trichrome) and collagen IV in the dermo-epidermal junction (anti-collagen IV antibody).

\section{RNA-seq analysis}

RNA-Seq analyses were narrowed to skin fragments from eight women due to sample quality control requirements. The broad differences among the studied areas were analyzed by principal component analysis (PCA). It was not possible to identify differences among the three areas (Fig. 2A) by PCA analysis, however, minor differences in gene expression were determined for each comparison. Comparison between A1 versus A2, exposed areas, revealed 88 differentiated expressed genes (DEGs) (51 down-regulated and 37 up-regulated). In addition, a substantial number of DEGs was detected when 
exposed and non-exposed areas were compared. For example, comparison between A1 versus A3 showed 554 DEGs (397 down-regulated and 157 up-regulated) and A2 versus A3 revealed 568 DEGs (336 down-regulated and 232 up-regulated). Overall, 917 DEGs were observed (Supplementary Table 1), most of them exclusively found in only one comparison. However, some overlap among the three comparisons was observed (Fig. 2B).

Since the PCA analysis containing the whole transcriptome expression data showed no clustering of samples sharing the same area source, two additional approaches were carried out. First one focused on the 917 DEGs wherein, a clear clustering between A1 and A2 might be appreciated from Fig. 2C. Similarly, the heat-map presented in Fig. 2D shows the hierarchical clustering based on gene expression among samples, wherein A3 samples (bottom of the figure) clustered together. The second PCA approach accounted for the overlap of A1 versus A3 and A2 versus A3 comparison DEGs (244). Like the previous analysis, it was possible to note the clustering in both PCA (Fig. 2E) and the heat-map (Fig. 2F).

Although, some similarities were found between A1 and A2 DEGs, the pathway analysis showed very different profile. While A1 versus A3 comparison predicted a decreased activation state of cellular movement, $A 2$ versus $A 3$ comparison predicted decreased activation pathways for cancer and other injuries in addition to activation of several lipid metabolism related pathways (Supplementary Table 2).

Comparison of $\mathrm{A} 2$ versus $\mathrm{A} 3$ focused on the differences between exposed and unexposed areas. Figure $3 \mathrm{~A}$ shows the volcano plot of $\mathrm{A} 2$ versus $\mathrm{A} 3$ comparison and distribution of DEGs. Figure $3 \mathrm{~B}$ shows the IPA diseases and function networks with a prediction of altered activation state together with the number of up and down-regulated genes. In addition, Fig. 3C, shows an overall picture of networks encompassed by DEGs for the A2 versus A3 comparison. It is possible to observe in the center a big orange portion containing increased activation state while, some sparse blue boxes are present all over the figure, showing decreased activation state networks.

\section{Discussion}

The study of skin aging mechanisms may be useful to understand the whole-body senescence. Therefore, epigenomic and transcriptomic analysis of aged skin from exposed and unexposed areas play important role in understanding human aging and age-related diseases. The photoaging is the major component of extrinsic aging [20]. The management of choice is the continuous use of broad-spectrum sunscreen [2] and topical retinoids, particularly all-trans retinoic acid or tretinoin, despite the risk of irritative dermatitis [46]. We have observed the increased skin roughness and deep wrinkles in photoexposed areas, compared to non-photoexposed.

Surprisingly no difference was detected in smoothness and scaling among areas. The low density of collagen IV in upper dermis in exposed areas is coincident with the findings of Caetano et al. (2016)[5]. So far, a well-documented therapeutic strategy for reversing the integrity of dermal matrix is the prolonged use of topical tretinoin [46]. However, the tolerability to tretinoin decreases with intrinsic aging as the skin becomes thinner and more sensitive, so other topical treatments are proposed. 
Targeting age-related lipid deficiency[25] and consequent stratum corneum dysfunction, may be a strategy in the development of therapies capable of increasing ceramides levels, improving cutaneous barrier function. Therefore, cosmeceuticals presenting efficacy comparable to retinoids in the dermis but also capable of modulating epidermis and stratum corneum are of high importance. Progresses have been made in this field based in nanotechnology and in vitro studies [24]. However, in vivo research is complimentary and relevant.

Mercurio et al., (2016)[32] and Carvalho et al., (2017)[9], described the biophysical properties of photoaged skin. Our findings corroborate to their reports, regarding skin roughness and wrinkles number, which were significantly higher in photo-exposed areas as consequence of extrinsic over intrinsic aging superposition. The periorbital area was smoother than preauricular, possibly related to better use of moisturizer around the eyes. The exposed skin presented more desquamation than the unexposed, representing the cutaneous barrier damage. Surprisingly, no differences were detected in the smoothness between preauricular and gluteal areas, due to regular use of moisturizer. The significantly higher echogenicity in $\mathrm{A} 1$ and $\mathrm{A} 2$ compared to $\mathrm{A} 3$ was not expected but can be explained by evident elastosis present in both photo-exposed areas and dermal alterations in the unexposed skin caused by intrinsic aging, such as reduction of extracellular matrix synthesis. In addition to elastosis, the low density of collagen fibers in the upper dermis, as well as the decreased epidermal thickness in exposed areas are consistent with the description published by Caetano et al. (2016)[5]. The digital quantitative analysis of histological findings revealed reduced epidermal thickness and flattened DEJ in periorbital compared to preauricular and gluteal skin. These aspects were already reported as consequence of ROS accumulation $[3,40]$, in addition to intrinsic aging mechanisms and repetitive muscular movement [31]. The papillary dermis of periorbital skin was thinner than preauricular and gluteal skin. The periorbital collagen fibers were also thinner with more irregular orientation and larger interfibrillar spaces. The level of collagen IV was lower than those of preauricular skin at DEJ. The collagen IV was considered a good marker for DEJ integrity by Raman microspectroscopy [34].

RNASeq data showed that cell death/survival pathways and lipid metabolism networks were enriched with a larger amount of DEGs with a predict impact by IPA analysis, suggesting they may play an important role in skin aging. As demonstrated by previous in vitro studies [43], we observed a downregulation of genes related to DNA replication and repair, cell cycle regulation and apoptosis as well as up-regulation of genes related to inflammatory response in photo-exposed areas ( $A 1$ vs $A 2$ ). This indicates that periorbital wrinkles seem to undergo a greater inflammatory damage than the photoexposed area without wrinkles.

The preauricular area was free of dynamic wrinkles, therefore the main aging factor would be sun exposure. In order to verify the photodamage, we compared the activated pathways in A2 versus A3. Interestingly, our results showed an increase in lipid and aminoacids metabolism in the preauricular when compared to gluteal area. The increased expression of lipid-related genes could be explained by the inflammatory process, as discussed by Zhuang and Lyga (2014)[48]. In the epidermis there is cellular damage with oxidized lipids that are capable of causing inflammation by activating the migration of 
macrophages to remove cells and oxidized lipids. Thus, macrophages overloaded with oxidized lipids release cytokines and ROS, causing inflammation and long-term damage also in the dermal matrix. Therefore, some genes have increased expression to compensate for lipids damaged. Skin suffers from both intrinsic and extrinsic factors of aging, however the increased expression of lipids in exposed skin could lead to more inflammation when compared to unexposed skin.

Shen et al. (2016)[43] demonstrated that UV genetic signature of skin was similar to squamous cell carcinoma. After UVB irradiation of human keratinocytes, they found 401 DEGs; the alterations were UVBdose dependent and persisted for 21 days. Downregulated genes were related to DNA replication and repair, cell cycle regulation, chromatin dynamic, and apoptosis. Additionally, upregulated genes were linked to inflammatory response and adaptive immunity. The decrease of epidermal lipids, such as triglycerides and free fatty acids, as well as the down regulation of genes related to lipid synthesis in aged skin was reported by Kim et al. (2010)[26]. The authors explained their findings by the increased expression of MMP-1, which is an inhibitor of lipid synthesis. They also suggested that moisturizer formulations similar to lipid composition of skin surface could restore the cutaneous barrier. The reduced expression of innate immunity markers in various cells during the aging process was more pronounced in sebaceous glands, pointing out a role of sebum in immune response which is compromised in elderly [18]. Authors concluded that the down-regulation of genes related to lipid metabolism could be explained by the inclusion of menopaused women who present reduced hormonal stimulation of sebaceous glands and keratinocytes, as reported by Calleja-Agius et al. (2007)[6]. However, our results were opposite, as several pathways related to lipid metabolism were predicted to be activate in photo-exposed skin without wrinkles. We could postulate this finding as a defense response to restore cutaneous barrier. The Genotype-Tissue Expression (GTEx) compared samples from photo-protected suprapubic and photodamaged lower leg areas from individuals of both genders, aged from 20 s to 70 s and skin tissue samples were obtained by rapid autopsy from donors; they created a database useful for comparison in skin genomic studies [13]. Cho et al. (2018)[10] analyzed RNA sequencing data from GTEx in order to identify transcriptomic changes of aged skin. The main results were the up-regulation of DEGs of epidermal differentiation complex component, vasculature development and matrix metalloproteinase in UV exposed area. The down-regulated DEGs included IL6 and IL33, involved in wound heading, and several histones. In contrast to our results, genes related to lipid metabolism were down-regulated in photo-damaged skin. This difference may be explained by the following reasons: GTEx used exposed skin from lower leg which is less damaged than the face; the samples were obtained from corpse and our study was in vivo; GTEx study population was from other geographic region, included a wide age range and both genders.

Kimball et al. (2018)[27] demonstrated divergent molecular processes along lifetime in skin affected by intrinsic (buttocks) and extrinsic (face and dorsal forearm) aging by analyzing epidermal gene expression. They included Caucasian females from six decades of age (20 to 74 yo.) who appear younger than their chronologic age. The pathways related to oxidative stress, energy metabolism, senescence, and epidermal barrier were accelerated in the 60s and 70s. Interestingly, gene expression 
patterns in women who were younger-appearing were similar to those in younger women, suggesting the major role of extrinsic aging.

A limitation of in vivo studies is the difficulty to separate epidermis and dermis to provide conclusive insights into intrinsic and extrinsic aging related gene expression in different skin levels and the respective cells. In vitro molecular analysis of cultured normal human dermal fibroblasts (NHDFs) isolated from intrinsically aged human skin from young subjects compared to middle aged and elderly donors have detected 998 proteins. From those, 70 were named skin aging-associated secreted proteins (SAASP) as they exhibited age-dependent secretion pattern. Their comparison with the senescenceassociated secretory phenotype (SASP) revealed common aspects related to matrix degradation and proinflammatory processes. On the other hand, 27 proteins, involved in metabolism and adherence junction, were specific for intrinsic aged skin dermal fibroblasts and possibly related to specific process[44]. Fibroblast from female breast skin, aged 20-30 and 60-70 years and keratinocytes from young foreskin were used to obtain a reconstructed human skin (RHS). Differences in gene expression including extracellular matrix proteins, growth factors and IL-6 were detected. The influence of fibroblasts in epidermal differentiation and stratum corneum lipid production was evident. Compared to young foreskin the stratum corneum was thicker in adult and aged RHS; E-cadherin levels were reduced and filaggrin expression was increased in adult and aged RHS; the amount of barrier lipids were also increased in adult and aged RHS[22].

It is extremely difficult to compare results from in vivo genomic studies as multiple parameters may lead

to differences in study population. However, coincident DEGs detected represent a relevant contribution to the knowledge of skin aging, in particular the role of environmental aggressors along with the study of networks predicted to be impacted according to gene expression profile. Our results add additional information about the role of sun exposure on skin aging process as well as help identify potential new targets for its control.

\section{Methods}

\section{Design}

Monocentric, cross-sectional and exploratory study. The Research Ethics Committee of Federal University of Sao Paulo approved this protocol (\#2.468.238). Informed consent was obtained from all subjects. All procedures were followed in accordance with ethical standards of the responsible committee on human experimentation (institutional and national) and with the Helsinki Declaration of 1975, as revised in 2013.

\section{Population and inclusion criteria}

Fifteen menopaused and healthy women, 55 to 65 yo, phototype III (Fitzpatrick's classification in 1988 [19]), with visible and moderate periorbital wrinkles at rest (Carruthers et al.'s classification in 2008 [8]) and visible signs of facial photoaging. The exclusion criteria included: use of topical and/or systemic medications (hormones, anti-coagulants, vasodilators, anti-platelet), which could interfere in the healing 
process; presence of abnormal facial scars; inflammatory, infectious, pre-neoplastic and neoplastic diseases on facial skin; history of allergic reactions to anesthetics; previous treatments or use of: topical tretinoin or oral isotretinoin (6 months), retinol, retinaldehyde, alfa-hydroxyacids, beta-hydroxyacid and ascorbic acid (3 months), facial procedures (ablative laser, intense pulsed light, dermabrasion, mediumdepth or deep chemical peeling, cryotherapy, photodynamic therapy (6 months), superficial chemical peeling, microdermabrasion, radiofrequency, microneedling, and non-ablative laser (3 months)), botulinum toxin or temporary filler (6 months), permanent filler (any time) and facial surgical lifting or blepharoplasty (any time); systemic diseases that could interfere in study activities or increase the risk of infections; alcohol or illegal drugs use and smoking.

Skin relief was assessed by VisioScan VC $98 \AA$ (Courage-Khazaka, Germany) and SELS (Surface Evaluation of Living Tissue) system represented by four quantitative and qualitative parameters: Skin Smoothness (SEsm), Skin Roughness (SEr), Scaliness (SEsc) and Wrinkles (SEw); total dermal thickness and upper dermis echogenicity by high frequency $(20 \mathrm{MHz})$ ultrasound (DermaScan®, Cortex Technology, Denmark)

\section{Sample collection and preparation}

Three $6 \mathrm{~mm}$-punch skin biopsies, after asepsis and local infiltrative anesthesia (10\% lidocaine with vasoconstrictor) were performed: A1 - periorbital wrinkles at rest, on right or left side (the most visible); A2- preauricular area at the same side as A1 and A3 - gluteal region. The suture line was placed in parallel to a normal skin line to become imperceptible. Each skin fragment was carefully bisected into two $3 \mathrm{~mm}$ pieces. One was immediately fixed in $10 \%$ buffered formalin and send to Pathology Laboratory to be included in paraffin blocks for production of slides. For histology, hematoxylin and eosin (HE) staining was used to evaluate the thickness and organization of epidermis and dermis and Masson's trichrome for dermal matrix collagen content. Immunohistochemistry, with biomarker for collagen IV, was used to evaluate basement membrane collagen in the DEJ. The slides were photographed and the images analyzed by the software Image $\mathrm{J}(\mathrm{NIH}, \mathrm{USA})$, which represents objective quantitative analysis, more precise than morphometric evaluation [45]. For statistical analysis of non-invasive measures (skin relief and dermal echogenicity), as well as histological and immunohistochemical findings, data were described by mean \pm standard deviation. For comparison between areas, mean \pm standard error, ONEWAYANOVA followed by multiple comparisons, with Bonferroni correction, were used. The $p$ value $<0.05$ was considered significant.

The second fragment was immediately frozen in liquid nitrogen and send to Molecular Biology Laboratory for gene expression analysis (RNAseq). The RNA was extracted by using QIAmpRNeasy Fibrous Tissue Mini Kit (Qiagen, CA). Skin fragments from only eight women were used, as the other contained insufficient material. Reverse transcribed RNA was prepared for each sample by Super Script III enzyme. Barcoded libraries were constructed using targeted regions from the human transcriptome. Each participant was identified by a different barcode with the lon Xpress ${ }^{\text {TM }}$ Barcode Adapters (Life Technologies, CA). 
Libraries, generated with lonPI ${ }^{\text {TM }} \mathrm{Hi}$ Q ${ }^{\text {TM }}$ OT 2200kitin One Touch2 TM Instrument, were sequenced using IonPITM Chipv3 at lonProtonTM Sequencer (Life Technologies,CA). Partek Flow (Parteklnc, MO) was used in order to assess gene expression data. Reads were mapped to Human Genome GRCh37 (HG19) using STAR 2.5.3 [15]. Gene counts were obtained by annotation model based on EnsemblTranscripts (release75) and normalized by counts per million (CPM) and by adding 0.0001 to all read counts, to allow statistical testing. Differential gene expression was determined by Partek GeneSetAnalysis (GSA) in the default settings.

Genes presenting fold-change higher than 2 or fold-change lower than -2 along with $p<0.05$ for each comparison were considered DEGs. Pathway analysis was performed using Ingenuity Pathway Analysis (IPA Qiagen, CA), where in enriched pathways were identified for each gene list from previously comparisons.

\section{Declarations}

Authors Contribution: RPM, PV, and RFSanalyze RNA-Seq data; RPM and PV drafted the manuscript; CPG and MMM conduced validation analysis experiments; SY and JLMS performed histological and immunohistochemistry experiments; JIB, SC, $\mathrm{CH}$, and $\mathrm{YZ}$, analyze histological and genetics data; AS perform statistical analysis; JL, JBP, and EB designed andsupervised the research; All authors revised and approved the final manuscript.

Funding: This study was funded by Avon Products Inc (NY, NY, 10901 Suffern, USA), Coordenação de Aperfeiçoamento de Pessoal de Nível Superior - Brasil (CAPES - Finance Code 001); Conselho Nacional de DesenvolvimentoCientífico e Tecnológico (CNPq), and Fundação de Amparo a Pesquisa do Estado de São Paulo (FAPESP 2014/27198-8).

Conflicts of Interest: RPM, PV, CPG, MMM, RFS, AS, SY, JLMS, JBP, and EB declare no conflict of interest. JIB, SC, CH, YZ, and JL work for Avon Products Inc (NY, NY, 10901 Suffern, USA). The funders had no role in the design of the study; in the collection, analyses, or interpretation of data; in the writing of the manuscript, or in the decision to publish the results.

Acknowledgments: We thank Dr. Gopi Menonfor his comments on the histology analysis of tissues relevant for this work.

\section{References}

1. Babamiri, K, Nassab, R (2010) Cosmeceuticals: the evidence behind the retinoids. Aesthet Surg J 30:74-7. https://doi.org/10.1177/1090820X09360704

2. Bens, G (2014) Sunscreens. Adv Exp Med Biol 810:429-63. https://doi.org/10.1007/978-1-4939-04372_25

3. Birch-Machin, MA, Russell, EV, Latimer, JA (2013) Mitochondrial DNA damage as a biomarker for ultraviolet radiation exposure and oxidative stress. Br J Dermatol 169 Suppl 2:9-14. 
https://doi.org/10.1111/bjd.12207

4. Brandt, FS, Cazzaniga, A, Hann, M (2011) Cosmeceuticals: current trends and market analysis. Semin Cutan Med Surg 30:141-3. https://doi.org/10.1016/j.sder.2011.05.006

5. Caetano, LdVN, Soares, JL, Bagatin, E, Miot, HA (2016) Reliable assessment of forearm photoageing by high-frequency ultrasound: a cross-sectional study. Int J Cosmet Sci 38:170-7. https://doi.org/10.1111/ics.12272

6. Calleja-Agius, J, Muscat-Baron, Y, Brincat, MP (2007) Skin ageing. Menopause Int 13:60-4. https://doi.org/10.1258/175404507780796325

7. Cao, C, Xiao, Z, Wu, Y, Ge, C (2020) Diet and Skin Aging-From the Perspective of Food Nutrition. Nutrients 12. https://doi.org/10.3390/nu12030870

8. Carruthers, A, Carruthers, J, Hardas, B, Kaur, M, Goertelmeyer, R, Jones, D, Rzany, B, Cohen, J, Kerscher, M, Flynn, TC, Maas, C, Sattler, G, Gebauer, A, Pooth, R, McClure, K, Simone-Korbel, U, Buchner, L (2008) A validated grading scale for crow's feet. Dermatol Surg 34 Suppl 2:S173-8.

https://doi.org/10.1111/j.1524-4725.2008.34367.x

9. Carvalho, PRS, Sumita, JM, Soares, JLM, Sanudo, A, Bagatin, E (2017) Forearm skin aging:

characterization by instrumental measurements. Int J Cosmet Sci 39:564-571. https://doi.org/10.1111/ics.12407

10. Cho, BA, Yoo, SK, Seo, JS (2018) Signatures of photo-aging and intrinsic aging in skin were revealed by transcriptome network analysis. Aging (Albany NY) 10:1609-1626.

https://doi.org/10.18632/aging.101496

11. Cho, S, Shin, MH, Kim, YK, Seo, JE, Lee, YM, Park, CH, Chung, JH (2009) Effects of infrared radiation and heat on human skin aging in vivo. J Investig Dermatol Symp Proc 14:15-9.

https://doi.org/10.1038/jidsymp.2009.7

12. Choi, EH (2019) Aging of the skin barrier. Clin Dermatol 37:336-345. https://doi.org/10.1016/j.clindermatol.2019.04.009

13. Consortium, GT (2015) Human genomics. The Genotype-Tissue Expression (GTEx) pilot analysis: multitissue gene regulation in humans. Science 348:648-60.

https://doi.org/10.1126/science. 1262110

14. de Diego, I, Peleg, S, Fuchs, B (2019) The role of lipids in aging-related metabolic changes. Chem Phys Lipids 222:59-69. https://doi.org/10.1016/j.chemphyslip.2019.05.005

15. Dobin, A, Davis, CA, Schlesinger, F, Drenkow, J, Zaleski, C, Jha, S, Batut, P, Chaisson, M, Gingeras, TR (2013) STAR: ultrafast universal RNA-seq aligner. Bioinformatics 29:15-21. https://doi.org/10.1093/bioinformatics/bts635

16. Draelos, ZD (2009) Cosmeceuticals: undefined, unclassified, and unregulated. Clin Dermatol 27:4314. https://doi.org/10.1016/j.clindermatol.2009.05.005

17. Draelos, ZD (2011) The art and science of new advances in cosmeceuticals. Clin Plast Surg 38:397407, vi. https://doi.org/10.1016/j.cps.2011.02.002 
18. Elewa, RM, Abdallah, MA, Zouboulis, CC (2015) Age-associated skin changes in innate immunity markers reflect a complex interaction between aging mechanisms in the sebaceous gland. $J$ Dermatol 42:467-76. https://doi.org/10.1111/1346-8138.12793

19. Fitzpatrick, TB (1988) The validity and practicality of sun-reactive skin types I through VI. Arch Dermatol 124:869-71. https://doi.org/10.1001/archderm.124.6.869

20. Flament, F, Bazin, R, Laquieze, S, Rubert, V, Simonpietri, E, Piot, B (2013) Effect of the sun on visible clinical signs of aging in Caucasian skin. Clin Cosmet Investig Dermatol 6:221-32. https://doi.org/10.2147/CCID.S44686

21. Franca, K, Cohen, JL, Grunebaum, L (2013) Cosmeceuticals for recurrence prevention after prior skin cancer: an overview. J Drugs Dermatol 12:516-8.

22. Hausmann, C, Zoschke, C, Wolff, C, Darvin, ME, Sochorova, M, Kovacik, A, Wanjiku, B, Schumacher, F, Tigges, J, Kleuser, B, Lademann, J, Fritsche, E, Vavrova, K, Ma, N, Schafer-Korting, M (2019) Fibroblast origin shapes tissue homeostasis, epidermal differentiation, and drug uptake. Sci Rep 9:2913. https://doi.org/10.1038/s41598-019-39770-6

23. Hughes, MC, Williams, GM, Baker, P, Green, AC (2013) Sunscreen and prevention of skin aging: a randomized trial. Ann Intern Med 158:781-90. https://doi.org/10.7326/0003-4819-158-11201306040-00002

24. Iqbal, B, Ali, J, Baboota, S (2018) Recent advances and development in epidermal and dermal drug deposition enhancement technology. Int J Dermatol 57:646-660. https://doi.org/10.1111/ijd.13902

25. Jonca, $N$ (2019) Ceramides metabolism and impaired epidermal barrier in cutaneous diseases and skin aging: focus on the role of the enzyme PNPLA1 in the synthesis of $\omega$-0-acylceramides and its pathophysiological involvement in some forms of congenital ichthyoses $₫$. OCL 26:17.

26. Kim, EJ, Jin, XJ, Kim, YK, Oh, IK, Kim, JE, Park, CH, Chung, JH (2010) UV decreases the synthesis of free fatty acids and triglycerides in the epidermis of human skin in vivo, contributing to development of skin photoaging. J Dermatol Sci 57:19-26. https://doi.org/10.1016/j.jdermsci.2009.10.008

27. Kimball, AB, Alora-Palli, MB, Tamura, M, Mullins, LA, Soh, C, Binder, RL, Houston, NA, Conley, ED, Tung, JY, Annunziata, NE, Bascom, CC, Isfort, RJ, Jarrold, BB, Kainkaryam, R, Rocchetta, HL, Swift, DD, Tiesman, JP, Toyama, K, Xu, J, Yan, X, Osborne, R (2018) Age-induced and photoinduced changes in gene expression profiles in facial skin of Caucasian females across 6 decades of age. J Am Acad Dermatol 78:29-39 e7. https://doi.org/10.1016/j.jaad.2017.09.012

28. Kohl, E, Steinbauer, J, Landthaler, M, Szeimies, RM (2011) Skin ageing. J Eur Acad Dermatol Venereol 25:873-84. https://doi.org/10.1111/j.1468-3083.2010.03963.x

29. Kolovou, GD, Bilianou, HG (2008) Influence of aging and menopause on lipids and lipoproteins in women. Angiology 59:54S-7S. https://doi.org/10.1177/0003319708319645

30. Lee, YM, Kim, YK, Chung, JH (2009) Increased expression of TRPV1 channel in intrinsically aged and photoaged human skin in vivo. Exp Dermatol 18:431-6. https://doi.org/10.1111/j.16000625.2008.00806.x 
31. Lighthall, JG (2018) Rejuvenation of the Upper Face and Brow: Neuromodulators and Fillers. Facial Plast Surg 34:119-127. https://doi.org/10.1055/s-0038-1637004

32. Mercurio, DG, Jdid, R, Morizot, F, Masson, P, Maia Campos, PM (2016) Morphological, structural and biophysical properties of French and Brazilian photoaged skin. Br J Dermatol 174:553-61. https://doi.org/10.1111/bjd.14280

33. Newburger, AE (2009) Cosmeceuticals: myths and misconceptions. Clin Dermatol 27:446-52. https://doi.org/10.1016/j.clindermatol.2009.05.008

34. Nguyen, TT, Gobinet, C, Feru, J, Pasco, SB, Manfait, M, Piot, O (2012) Characterization of Type I and IV Collagens by Raman Microspectroscopy: Identification of Spectral Markers of the DermoEpidermal Junction. Spectroscopy: An International Journal 27:686183. https://doi.org/10.1155/2012/686183

35. Nkengne, A, Bertin, C (2013) Aging and facial changes-documenting clinical signs, part 1: clinical changes of the aging face. Skinmed 11:281-6.

36. Oyewole, AO, Birch-Machin, MA (2015) Sebum, inflammasomes and the skin: current concepts and future perspective. Exp Dermatol 24:651-4. https://doi.org/10.1111/exd.12774

37. Palmer, DM, Kitchin, JS (2010) Oxidative damage, skin aging, antioxidants and a novel antioxidant rating system. J Drugs Dermatol 9:11-5.

38. Papsdorf, K, Brunet, A (2019) Linking Lipid Metabolism to Chromatin Regulation in Aging. Trends Cell Biol 29:97-116. https://doi.org/10.1016/j.tcb.2018.09.004

39. Rahimpour, Y, Hamishehkar, H (2012) Liposomes in cosmeceutics. Expert Opin Drug Deliv 9:443-55. https://doi.org/10.1517/17425247.2012.666968

40. Rinnerthaler, M, Bischof, J, Streubel, MK, Trost, A, Richter, K (2015) Oxidative stress in aging human skin. Biomolecules 5:545-89. https://doi.org/10.3390/biom5020545

41. Rogers, J, Harding, C, Mayo, A, Banks, J, Rawlings, A (1996) Stratum corneum lipids: the effect of ageing and the seasons. Arch Dermatol Res 288:765-70. https://doi.org/10.1007/BF02505294

42. Shanbhag, S, Nayak, A, Narayan, R, Nayak, UY (2019) Anti-aging and Sunscreens: Paradigm Shift in Cosmetics. Adv Pharm Bull 9:348-359. https://doi.org/10.15171/apb.2019.042

43. Shen, Y, Kim, AL, Du, R, Liu, L (2016) Transcriptome Analysis Identifies the Dysregulation of Ultraviolet Target Genes in Human Skin Cancers. PLoS One 11:e0163054. https://doi.org/10.1371/journal.pone.0163054

44. Waldera Lupa, DM, Kalfalah, F, Safferling, K, Boukamp, P, Poschmann, G, Volpi, E, Gotz-Rosch, C, Bernerd, F, Haag, L, Huebenthal, U, Fritsche, E, Boege, F, Grabe, N, Tigges, J, Stuhler, K, Krutmann, J (2015) Characterization of Skin Aging-Associated Secreted Proteins (SAASP) Produced by Dermal Fibroblasts Isolated from Intrinsically Aged Human Skin. J Invest Dermatol 135:1954-1968. https://doi.org/10.1038/jid.2015.120

45. Wolf, DE, Samarasekera, C, Swedlow, JR (2007) Quantitative analysis of digital microscope images. Methods Cell Biol 81:365-96. https://doi.org/10.1016/S0091-679X(06)81017-4 
46. Zasada, M, Budzisz, E (2019) Retinoids: active molecules influencing skin structure formation in cosmetic and dermatological treatments. Postepy Dermatol Alergol 36:392-397. https://doi.org/10.5114/ada.2019.87443

47. Zhang, S, Duan, E (2018) Fighting against Skin Aging: The Way from Bench to Bedside. Cell Transplant 27:729-738. https://doi.org/10.1177/0963689717725755

48. Zhuang, Y, Lyga, J (2014) Inflammaging in skin and other tissues - the roles of complement system and macrophage. Inflamm Allergy Drug Targets 13:153-61. https://doi.org/10.2174/1871528113666140522112003

\section{Figures}
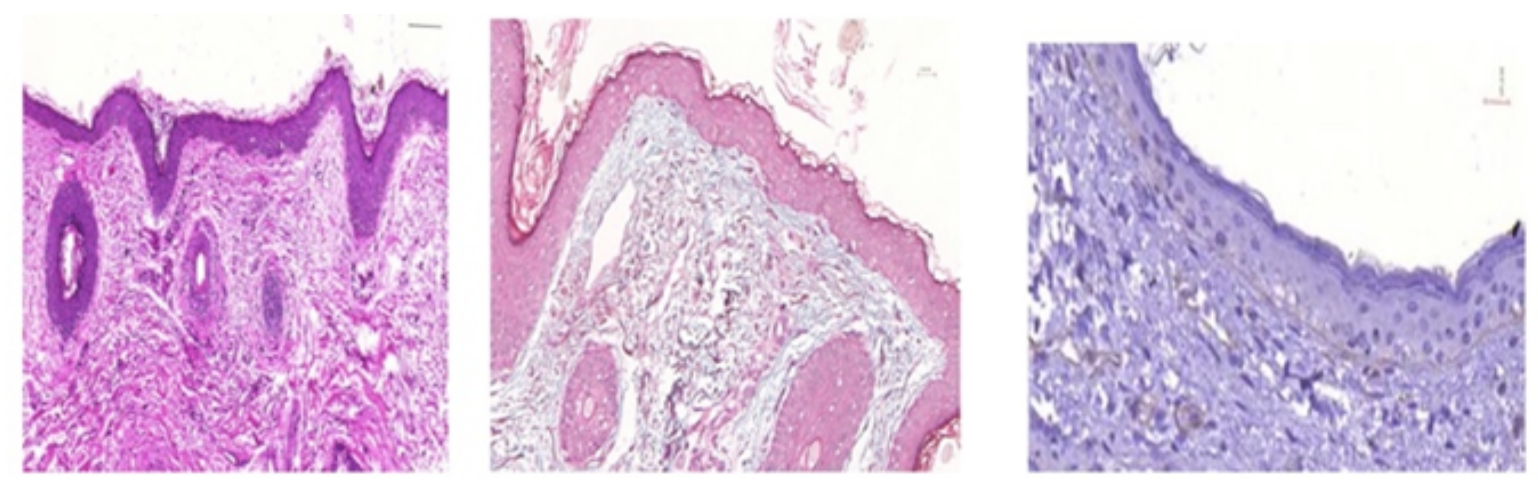

Periorbital (crow's feet)

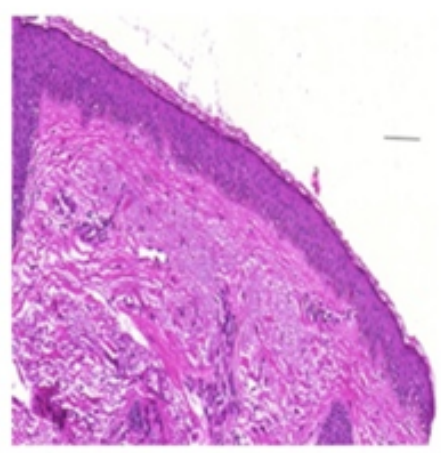

(A) $H \& E$

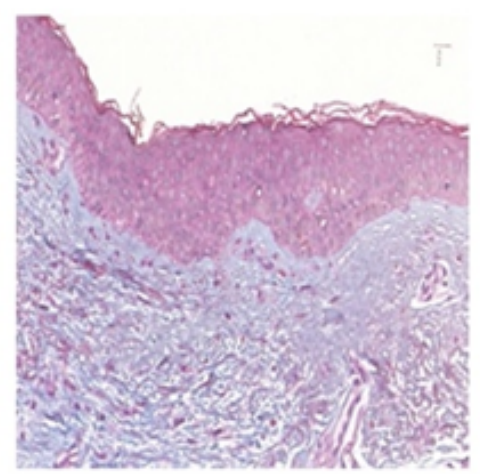

(B) Masson's trichrome

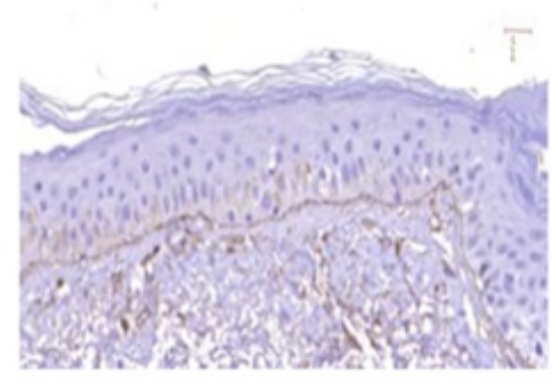

Preauricular (wrinkle-free)

\section{Figure 1}

Histological and immunohistochemical findings from periorbital and preauricular skin fragments stained with (A) Hematoxylin and Eosin (H\&E), (B) Masson's trichrome and (C) anti-collagen IV antibody. 
A

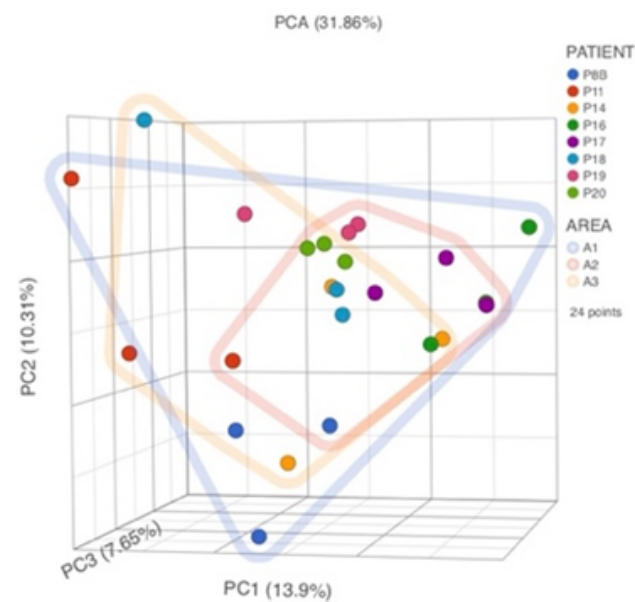

B
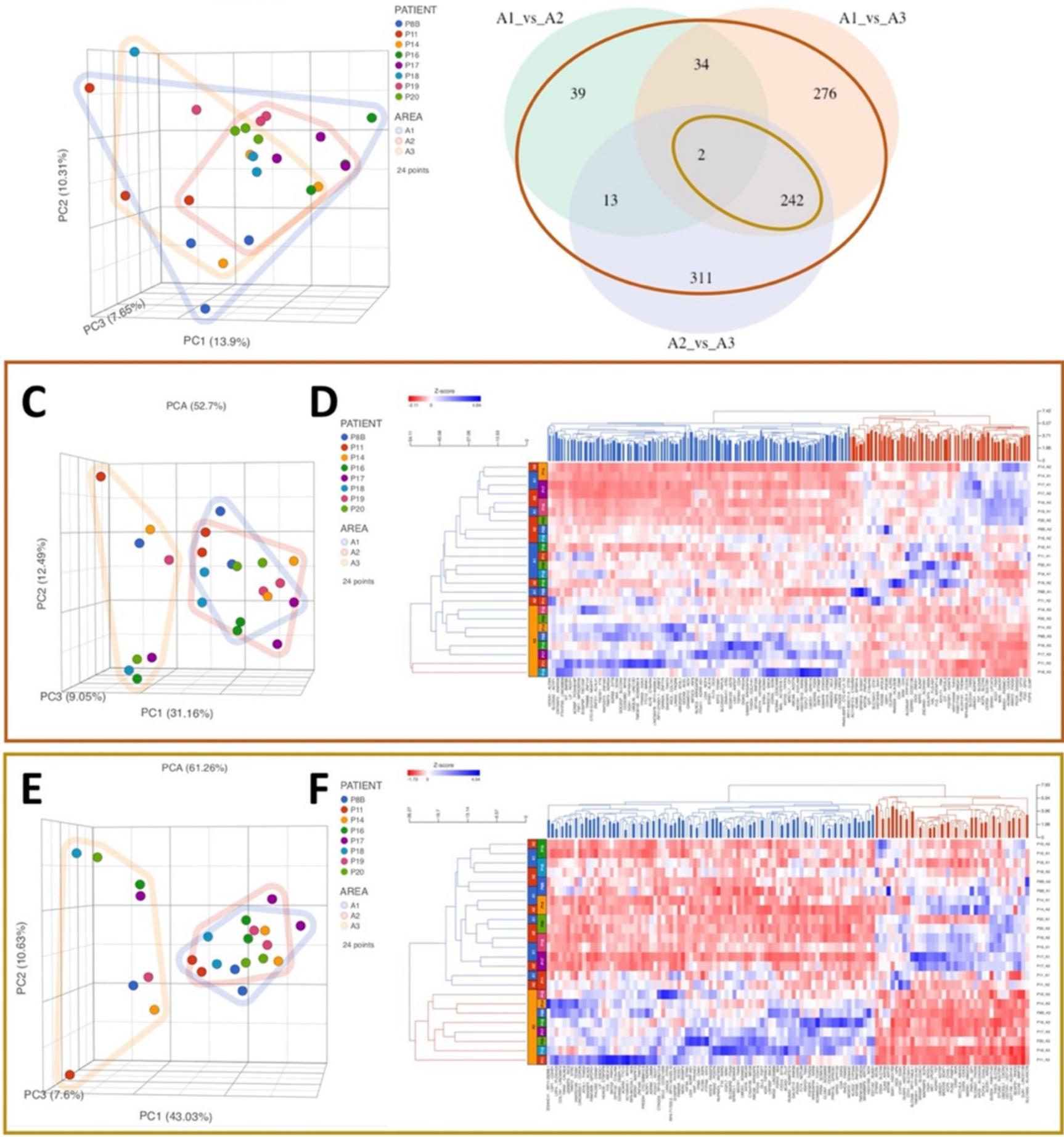

Figure 2

Gene expression analysis. Panel A, PCA analysis. Panel B, Venn diagram of differentially expressed genes in each of the comparisons along with their overlapping. Panel C, PCA analysis of all 917 Differentially Expressed genes. Panel D, hierarchical clustering of all 917 Differentially Expressed genes. Panel E, PCA analysis of 244 genes that overlap between $A 1$ versus $A 3$ comparison and $A 2$ versus $A 3$ comparison. Panel F, hierarchical clustering of 244 genes that overlap between A1 versus A3 comparison and A2 
versus A3 comparison. A1 - Periorbital area (crow's feet); A2 - Preauricular area (wrinkle-free); A3 Gluteal area.

\section{A}

B

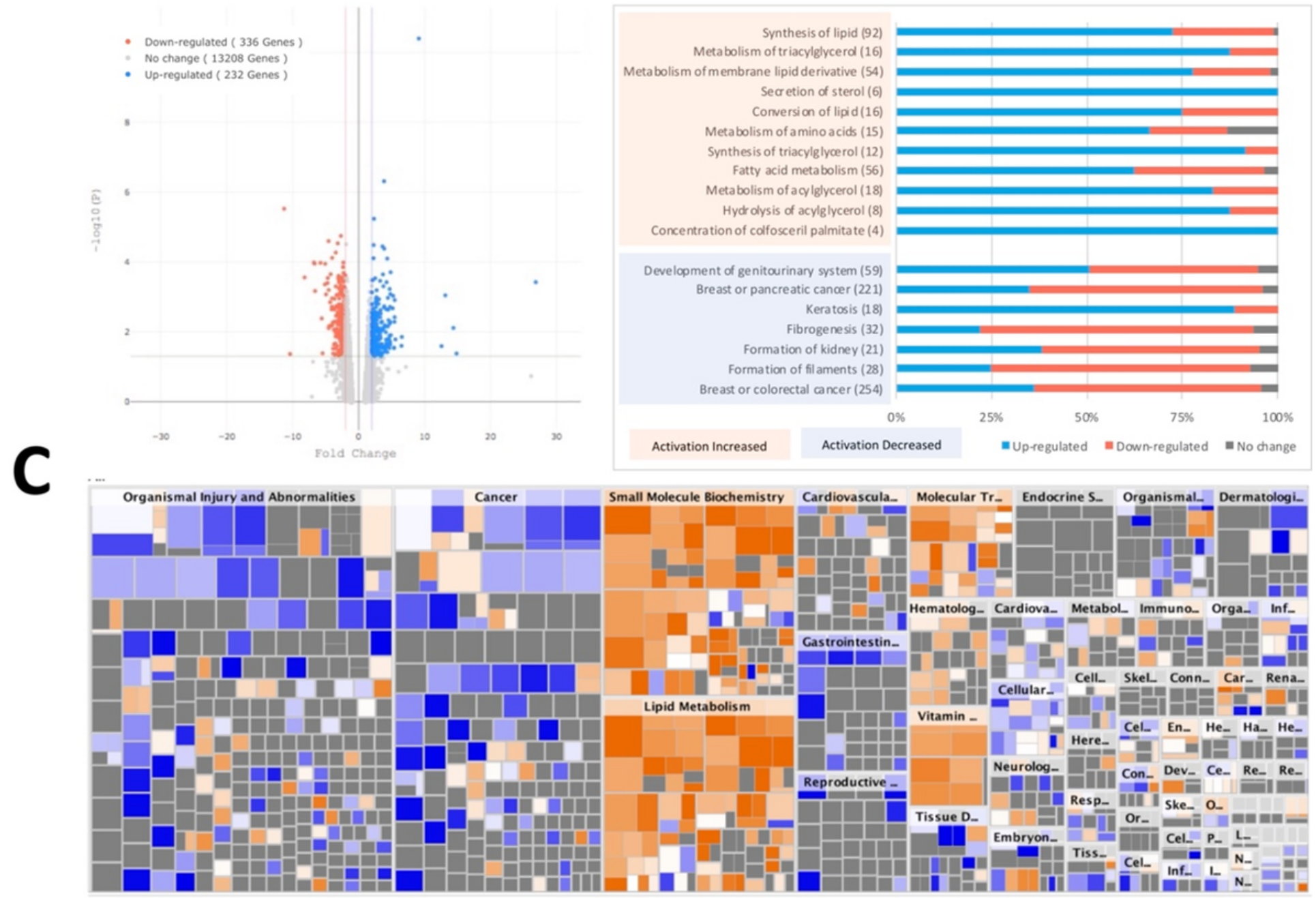

\section{Figure 3}

Pathway analysis of A2 versus A3 comparison. Panel A, Volcano plot showing Differentially Expressed Genes found on A2 versus A3 comparison. Panel B, impacted networks based on Differentially Expressed Genes found on A2 versus A3 comparison. Network (number of genes). Bars show percentage of downregulated, up-regulated, and no change genes in impacted network. Panel C, Overall picture of impacted of impacted networks, square sized by -Log(p-value) and square colored by Z-score. Blue Z-score $<0$ with decreased activation state, Orange Z-score $>0$ with increased activation state, and Gray no change on activation state.

\section{Supplementary Files}

This is a list of supplementary files associated with this preprint. Click to download. 
- PaperMartin2021SuppTable1.xlsx

- PaperMartin2021SuppTable2.xlsx 